\title{
Об одной задаче оптимизации конструкций с учетом требований устойчивости, прочности, при ограничениях первой частоты собственных колебаний
}

\author{
Л.С.Ляхович, ТГАСУ, Томск \\ П.А.Акимов, НИУ МГСУ, Москва \\ Б.А.Тухфатуллин, ТГАСУ, Томск
}

В одной из предыдущих работ авторов данной статьи рассматривалась задача оценки близости решения к проекту минимальной материалоёмкости при оптимизации ширины полок стержней двутаврового поперечного сечения при ограничениях по устойчивости или величины первой частоты собственных колебаний с учётом требований прочности в случае непрерывного изменения варьируемых параметров по длине стержня. При этом известно, что в строительной практике в основном стержни проектируются с кусочно-постоянным изменением параметров сечения. В другой статье авторов был сформулирован критерий оценки оптимальных решений при формирования кусочно-постоянных участков полки стержней двутаврового сечения при ограничениях по устойчивости или величине первой частоты собственных колебаний, но без учёта требований прочности. В данной статье рассматривается задача оценки оптимальных решений при формировании кусочно-постоянных участков ширины полок стержней двутаврового сечения при ограничениях по устойчивости или величины первой частоты собственных колебаний, но с учётом требований прочности и некоторых конструктивных ограничений.

Ключевые слова: критерий, оптимизация, устойчивость, частота, критическая сила, формы потери устойчивости, формы собственных колебаний, приведённые напряжения, кусочно-постоянное изменение параметров, минимальная материалоемкость.

On One Problem of Optimization of Structures Considering the Requirements of Stability and Strength Under the Constraints of the First Frequency of Natural Vibrations

L.S.Lyakhovich, TSUAB, Tomsk

P.A.Akimov, MGSU, Moscow

B.A.Tukhfatullin, TSUAB, Tomsk

"Данная статья продолжает тему, начатую авторами в статьях «Критерии оценки оптимальных решений при формировании стержней с кусочно-постоянным изменением поперечных сечений при ограничениях по устойчивости или на величину первой собственной частоты. Часть 1: Теоретические основы» и «Критерии оценки оптимальных решений при формировании стержней с кусочно-постоянным изменением поперечных сечений при ограничениях по устойчивости или на величину первой собственной частоты. Часть 2: Примеры расчёта», опубликованных в 2019 году в международном научном журнале "International Journal for Computational Civil and Structural Engineering (Международный журнал по расчету гражданских и строительных конструкций), Volume 15, Issue 4.
In a previous paper the authors of this paper considered the problem of assessing the proximity of the decision to draft minimum material consumption by optimizing the width of the shelves bars beam cross-section subject to the limits of stability or values of the first natural frequency and the requirements of strength in the case of continuous changes of variable parameters along thelength of the rod. In construction practice, as a rule, rods are designed with a piecewise constant change in the size of the section. Previously, the authors formulated a criterion for evaluating optimal solutions for the formation of piecewise constant sections of the I-beam shelf with restrictions on stability or the value of the first frequency of natural vibrations, while the strength of the rod was assumed to be secured. This article clarifies the criterion for the case when the strength requirements are considered in the optimization process.

Keywords: criterion, optimization, stability, frequency, critical force, forms of stabilityloss, forms of natural vibrations, reduced stresses, piecewise constant change of parameters, minimum material consumption.

\section{1. Введение, постановка задач исследования}

При решении оптимизационных задач [1-10] часто за признак достижения экстремума принимаются достаточно малые изменения функции цели на этапах вычислений. Известны случаи, когда этот признак достижения экстремума приводит к решению, которое значительно отличается от оптимального. Данное обстоятельство подтверждает актуальность работ, в которых вместе с анализом изменений в процессе вычислений приращений функции цели формулируются и используются критерии, оценивающие близость полученных решений к оптимуму. В частности, в [11-13] рассматривалась задача о оптимизации очертания

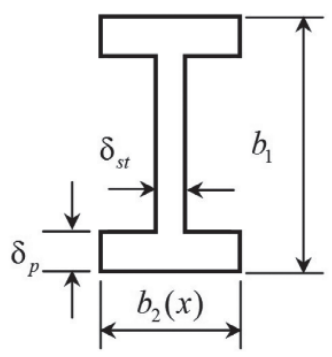

Рис. 1. Обозначения основных размеров рассматриваемого сечения 
ширины полок стержней двутаврового поперечного сечения стержня, загруженного продольной силой и несущего распределённую массу, при ограничениях по устойчивости или на величину первой частоты собственных колебаний. Обозначения размеров сечения приведены на рисунке 1 .

В процессе оптимизации высота сечения $b_{1}$, толщина стенки $\delta_{\text {st }}$, толщина полки $\delta_{\mathrm{p}}$ не варьируются. Варьируется функция $b_{2}(\mathrm{x})$ изменения ширины полок.

Функция цели записывается в виде:

$$
V_{0}=2 \int_{0}^{l} b_{2}(x) \delta_{p} d x
$$

или при дискретной модели стержня из п участков:

$$
V_{0}=2 \frac{l}{n} \sum_{1}^{n} b_{2}[i] \delta_{p} \text {. }
$$

Ограничение по устойчивости записывается в виде:

$$
P \leq P_{\mathrm{kp}}
$$

а на величину первой частоты собственных колебаний в виде:

$$
\omega_{0} \leq \omega_{1^{\prime}}
$$

где $V_{0}$ - объём материала полки; $P$ - действующая сила; $P_{k p}$ - критическая сила стержня; $\omega_{0}$ - заданная величина; $\omega_{1}-$ значение первой частоты собственных колебаний системы.

Требуется отыскать функцию $b_{2}(x)$, доставляющую минимум объёму материала полок $V_{0}$ при условии выполнения ограничений (3) и (4). Решение этой задачи может быть выполнено одним из известных методов оптимизации.

В упомянутых выше работах [11-13] а также в статьях [15; 16] сформулированы критерии, оценивающие близость полученных оптимизационных решений представленной выше задачи к оптимуму. В [11] предложен критерий, который позволяет оценить близость полученного оптимизационного решения к минимально материалоёмкому проекту. Один из вариантов записи критерия представлен в виде:

$\bar{\sigma}_{1 \omega t}^{2}(x)=\sigma_{1 \omega}^{2}(x) b_{1}-\sigma_{1 \omega t}^{2}(x)\left(b_{1}-2 \delta_{p}\right)-6 E\left(\omega_{0}\right)^{2} \rho \delta_{p} v_{\omega}^{2}(x)=$ const ,

где $\sigma_{1 \omega}(x)$ и $\sigma_{1 \omega t}(x)$ - соответственно нормальные напряжения в крайних волокнах двутаврового сечения и в волокнах на границе стенки и полки, вызываемые изгибающими моментами, возникающими при потере устойчивости или собственных колебаниях; $E$ - модуль упругости материала стержня; $\rho$ удельная масса; $v_{\omega}(x)$ - ординаты формы потери устойчивости или собственных колебаний.

Критерий (5) сформулирован при условии, что ширина полки $b_{2}(x)$ двутавра изменяется непрерывно по длине стержня.

Как известно, во многих практически важных случаях конструкторы предпочитают кусочно-постоянное изменение варьируемой величины.

В работе [12] сформулирован критерий, позволяющий оценивать результаты решений об оптимизации ширины полки при ограничениях по устойчивости или величины первой частоты собственных колебаний, но при кусочно-постоянном изменении размеров $b_{2}[i], i=1,2, \ldots, n$, где $n-$ количество участков кусочно-постоянного изменения ширины полки. Длины участков изменения полки $l_{u}[i]$, координаты правых краёв участков $x[i]$, левого края стержня $x$ [0] считаются заданными. Один из вариантов записи критерия представлен в виде:

$S_{1}[i]=\frac{1}{l_{k}[i]} \int_{x[i-1]}^{x[i]}\left[\sigma_{1 \omega}^{2}(x) b_{1}-\sigma_{1 \omega t}^{2}(x)\left(b_{1}-2 \delta_{p}\right)-6 E \omega_{0}^{2} \rho \delta_{p} v_{\omega}^{2}\right] d x=$ const

Задачи, для которых сформулированы критерии в [11; 12], не учитывали условий прочности. Поэтому полученные при этом оптимизационные решения могли этим условиям не удовлетворять. Если после оптимизации изменить полученный проект так, чтобы выполнялись ранее не учтённые в ходе оптимизации ограничения, то условия оптимальности будут нарушены.

В [13] предлагается учитывать в процессе оптимизации очертания полок, непрерывно меняющихся по длине стержня двутаврового сечения, кроме ограничений по устойчивости или величины первой частоты собственных колебаний, ещё и условия прочности, а также некоторые конструктивные ограничения.

Функция цели также записывается в виде (1), а при использовании дискретной модели в виде (2). Ограничения по устойчивости записываются в виде (3), а на величину первой частоты собственных колебаний в виде (4). Для оценки выполнения ограничений (3), (4) используется критерий (5).

Как известно, для стержней двутаврового поперечного сечения должны выполняться три условия прочности².

Для крайних волокон ограничение на нормальные напряжения:

$$
\sigma(x) \leq R,
$$

где $\sigma(x)$ - нормальные напряжения от нагрузки в крайних волокнах стержня; $R$ - расчётное сопротивление материала конструкции.

Для места стыка полки со стенкой:

$$
\sigma_{\text {экв }}(x)=\sqrt{\sigma_{p}^{2}(x)+3 \tau_{p}^{2}(x)} \leq R,
$$

где $\sigma_{\text {экв }}(x), \sigma_{\mathrm{p}}(x), \tau_{\mathrm{p}}(x)$ - соответственно эквивалентные, нормальные и касательные напряжения от нагрузки в месте стыка полки со стенкой.

Для уровня центра тяжести сечения:

$$
\sigma_{\text {экв }}(x)=\sqrt{\sigma_{0}^{2}(x)+3 \tau_{0}^{2}(x)} \leq R,
$$

где $\sigma_{\text {экв }}(x), \sigma_{0}(x), \tau_{0}(x)$ - соответственно эквивалентные, нормальные и касательные напряжение от нагрузки в центре тяжести сечения.

Для условий (8) и (9) использована четвёртая теория прочности.

${ }^{2}$ СП 16.13330.2017 Стальные конструкции» (актуализированная редакция СНиП II-23-81*). - М. : Минстрой России, 2017. - 140 с. 
Конструктивные ограничения на ширину полки в [13] приняты в виде:

$$
b_{2}(x) \geq b_{\text {min }}
$$

где $b_{\min }$ - минимально допустимые размеры ширины полки.

При использовании дискретной модели стержня соответствующие координаты сечения $(x)$ заменяются номером участка модели [i].

Для единообразия использования критериев и ограничений для оценки близости полученного в результате оптимизации проекта к минимально материалоёмкому критерии (5) и (6), условия (7), (8), (9) и (10) нормированы так, чтобы при их выполнении в виде равенств они бы принимали величину равную единице.

Для нормирования критерия (5) или (6) изгибающие моменты определяются по форме потери устойчивости или по первой форме собственных колебаний с точностью до постоянного множителя. По найденным моментам в сечениях стержня вычисляются напряжения $\sigma_{1 \omega}(x), \sigma_{1 \omega t}(x), \sigma_{1 \omega t}^{-2}(x)$. Затем среди значений $\sigma_{1 \omega t}^{-2}(x)$ выбирается наибольшее и на него делятся величины, вычисленные по (5) или по (6).

Теперь, если полученный в результате оптимизации проект является минимально материалоёмким, то критерий принимает вид:

$$
\sigma_{1 \omega \mathrm{t}}^{-2}(x) \leq 1 .
$$

Если при оптимизации использовались ещё и ограничения (7)-(10), то критерий (11) относится только к тем участкам стержня, на которых зависимости (7)-(10) выполняются в виде строгих неравенств.

Нормирование ограничений (7)-(9) выполняется делением обеих частей соответствующих неравенств на величину $R$ :

$$
\begin{gathered}
R_{1}(x)=\frac{\sigma(x)}{R} \leq 1 ; \\
R_{2}(x)=\frac{\sigma_{\text {эКв }}(x)}{R}=\frac{\sqrt{\sigma_{p}^{2}(x)+3 \tau_{p}^{2}(x)}}{R} \leq 1 ; \\
R_{3}(x)=\frac{\sigma_{э К B}(x)}{R}=\frac{\sqrt{\sigma_{0}^{2}(x)+3 \tau_{0}^{2}(x)}}{R} \leq 1 .
\end{gathered}
$$

Для нормирования ограничения (10) обе части его выражения делятся на $b_{2}(x)$ и записываются в виде:

$$
b_{0, \text { min }}(x)=\frac{b_{\min }}{b_{2}(x)} \leq 1 .
$$

В [13] через $\bar{\sigma}_{1 \text { юे }}^{2}(x), R_{1}(x), R_{2}(x), R_{3}(x)$ и $b_{0, \min }(x)$ обозначены соответственно показатели выполнения ограничений (3), (4), (7)-(10).

В соответствии с [13] при непрерывном изменении варьируемого параметра $b_{2}(x)$ близость кминимальной материалоёмкости проекта, полученного в результате оптимизации с учётом ограничений по устойчивости (или величине первой частоты собственных колебаний), ограничений по прочности и конструктивных, определяется близостью кединице обязательно в каждом сечении хотя бы одного из показателей (11)-(15).
Как известно, во многих практически важных случаях предпочитается кусочно-постоянное изменение варьируемой величины.

В данной статье обобщаются результаты [12; 13] на случай кусочно-линейного изменения варьируемого параметра ширины полок $b_{2}[i]$.

\section{2. Критерий оптимизации}

Рассматривается задача оценки близости к проекту минимальной материалоёмкости оптимальных решений при кусочно-постоянных участках ширины полок стержней двутаврового сечения и ограничениях по устойчивости или величине первой частоты собственных колебаний, но с учётом требований прочности и некоторых конструктивных ограничений.

Выбор границ кусочно-постоянных участков изменения ширины полки определяется как технологическими требованиями, так и стремлением приблизиться к минимально материалоёмкому решению к проекту при принятых ограничениях, но при непрерывном изменении $b_{2}[i]$ по длине стержня. После выбора границ участков размеры ширины полки определяются для каждого участка одним из методов оптимизации.

Для оценки близости этого решения к проекту минимальной материалоёмкости при выбранных границах участков используем показатели выполнения ограничений по прочности (12)-(14), конструктивные ограничения (15), а вместо показателя (11) нормированный к единице критерий (6), который запишется в виде:

$$
S_{1}(x) \leq 1
$$

Как известно [например [11] и СП 16.13330.2017. Стальные конструкции» (актуализированная редакция СНиП II-23$\left.81^{*}\right)$ ], если в задаче оптимизации некоторые ограничения выполняются в виде равенств, то они относятся к активным, а если в виде неравенств, то к пассивным. По аналогии назовём показатели выполнения ограничений, величина которых близка к единице, активными, а показатели, не отвечающие принятым условиям близости, пассивными.

Достаточность близости показателей выполнения ограничений к единице устанавливается конструктором и может характеризоваться разностью между единицей и соответствующим показателем.

Показатель (16) вычисляется в пределах каждого участка как определённый интеграл. Поэтому его величина относится ко всему участку.

Поскольку на каждом участке ширина полки неизменна, то показатель (15) для всех сечений соответствующего участка одинаков.

Так как изгибающие моменты и поперечные силы, возникающие в стержне от нагрузки, изменяются по длине стержня, то и в сечениях между границами участков показатели (12)-(14) будут различаться друг от друга. 
Если показатель (15) или (16) на участке достаточно близок к единице, то он на этом участке активный. Остальные показатели во всех сечениях этого участка должны выполняться в виде нестрогих неравенств.

Если показатель (12) или (13) или (14) на каком либо участке хотя бы в одном из сечений близок к единице, то он на этом участке активный. По ограничению, соответствующему этому показателю, определяется ширина полки. Несмотря на то что активный показатель относится к одному сечению, но найденная по активному ограничению ширина полки определяет величину ширины полки во всех сечениях этого участка. Остальные показатели во всех сечениях этого участка должны выполняться в виде нестрогих неравенств.

Сформулируем положение, определяющее близость проекта оптимизации в рассматриваемой задаче к проекту минимальной материалоёмкости:

«Проект оптимизации ширины полки при выбранных границах кусочно-постоянных участков стержней двутаврового поперечного сечения и ограничениях по устойчивости или на величину первой частоты собственных колебаний, ограничениях по прочности и конструктивных будет достаточно близок к минимально материалоёмкому, если на каждом участке хотя бы один из показателей (12)-(16) будет активным.

\section{3. Пример расчёта}

Проиллюстрируем изложенное положение на примере.

В [13] рассмотрен пример оптимизации ширины полок стержня двутаврового поперечного сечения при ограничениях на величину первой частоты собственных колебаний, ограничениях по прочности и конструктивных, но при непрерывном изменении варьируемого параметра $b_{2}[i]$ по длине стержня. Для сопоставления результатов оптимизации при непрерывном и кусочно-постоянном изменении варьируемого параметра $b_{2}[i]$ рассмотрим тот же пример. Схема стержня приведена на рисунке 2 а.

Высота сечения стержня $b=0,16$ м, толщина стенки $b_{\mathrm{st}}=$ 0,01 м, толщина полки $b_{\mathrm{p}}=0,014$ м.

Модуль упругости материала стержня $E=206000000000$

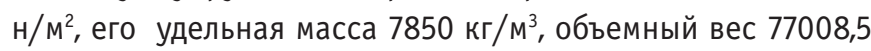
н/м³ расчётное сопротивление материала $R=240000000$ н/

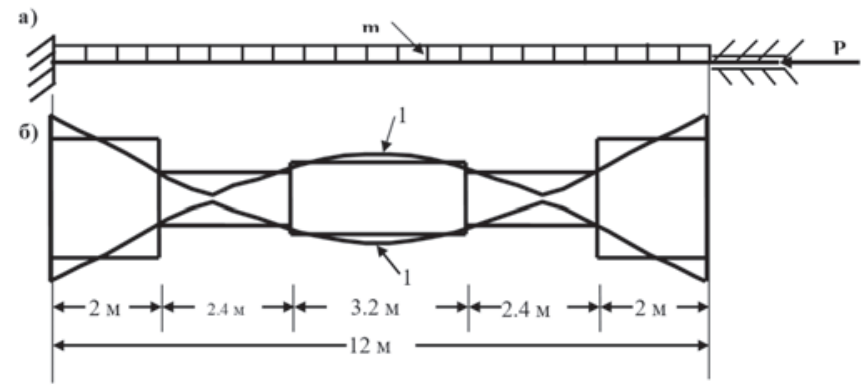

Pис. 2. Пример расчёта: а) схема стержня; б) очертание ширины полок
$M^{2}$. Стержень несёт равномерно распределённую массу интенсивностью $m=400$ кг/м. Эта же масса является внешней нагрузкой интенсивностью $q=3924$ н/м. При оптимизации с учётом условий прочности учитывается и собственный вес стержня.

Требуется оптимизировать значения ширины полок рассматриваемого стержня по участкам при $P=300000$ н, обеспечить величину первой частоты собственных колебаний $\omega_{1}$ $\geq \omega_{0}=13$ сек $^{-1}$ и минимальный объём материала полок. При оптимизации необходимо учитывать ограничения на величину первой частоты собственных колебаний (4), ограничения по прочности (7)-(9) и конструктивные (10). Примем также, как и в [13] минимально возможную ширину полки $b_{\min }=0,01$ м.

Расчёты выполняются на основе дискретной модели стержня из 30 участков. Равномерно распределённая масса и нагрузка приведена к узлам. В дискретной модели имеем узловые массы $m[i]=160$ кг и нагрузки $q[i]=1569,6$ н. Масса и вес конструкции учитываются в процессе оптимизации. Оптимизация выполнялась методом случайного поиска. Результаты представлены в таблице. В первом столбце таблицы представлены номера сечений дискретной модели (i). Во втором столбце приведены размеры ширины полок, полученные в результате оптимизации при непрерывном изменении варьируемого параметра $b_{2}[i]$. На рисунке 26 очертание ширины полок показано линиями 1.

Данный пример приводится для иллюстрации возможности использования показателей выполнения ограничений (12)-(16) для оценки оптимального решения при кусочно-постоянном изменении ширины полки. Поэтому для этой цели ограничимся одним вариантом выбора границ участков. В примере принят вариант пяти участков.

Как уже отмечалось, выбор границ кусочно-постоянных участков изменения ширины полки определяется как технологическими требованиями, так и стремлением максимально приблизиться к минимально материалоёмкому решению при непрерывном изменении $b_{2}[i]$ по длине стержня. Выбранные границы участков показаны на рисунке 2. При выбранных границах участков оптимальные размеры ширины полки определялись методом случайного поиска. Результаты вычислений показаны в столбце 3 таблицы 1. В столбце 9 показаны номера участков постоянной жёсткости $(k)$. В столбцах 4-8 приведены с точностью до четырёх знаков после запятой значения показателей оценки выполнения ограничений.

Показатель (15) $-b_{0, \min }[k]$, оказался пассивным на всех участках. Он выполняется для всех сечений в виде неравенств.

Показатель (16) - $S_{1}[k]$, оказался активным на первом, третьем и пятом участках. На втором и четвёртом участках он выполняется в виде неравенств.

Показатель (12) - $R_{1}[i]$, оказался активным в сечении 11 второго участка и в сечении 20 четвёртого участка. В остальных сечениях стержня показатель (12) выполняется в виде неравенств. 
Показатели $R_{2}[i], R_{3}[i]$ во всех сечениях стержня выполняются в виде неравенств. В таблице активные показатели выделены.

Итак, в данном примере на каждом участке есть по одному активному показателю. При этом остальные показатели также выполняются, но в виде неравенств.

Следовательно, результат оптимизации близок к минимально материалоёмкому проекту при выбранных для рассмотренного примера границах участков.
Таким образом, в настоящей статье сформулировано и верифицировано теоретическое положение об оценке близости проекта оптимизации ширины полки при выбранных границах кусочно-постоянных участков стержней двутаврового поперечного сечения и ограничениях по устойчивости или на величину первой частоты собственных колебаний с учётом ограничений по прочности и конструктивных требований к проекту минимальной материалоёмкости.

Таблица 1. Результаты расчета

\begin{tabular}{|c|c|c|c|c|c|c|c|c|}
\hline \multirow[t]{2}{*}{$i$} & \multicolumn{2}{|c|}{$b_{2}[i]$} & \multicolumn{5}{|c|}{ Показатели оценки } & \multirow[t]{2}{*}{ k } \\
\hline & & & $R_{1}[i]$ & $R_{2}[i]$ & $R_{3}[\mathrm{i}]$ & $b_{0, \min }[i]$ & $S_{1}[k]$ & \\
\hline 1 & 0,1995 & 0,1492 & 0,9871 & 0,8633 & 0,2685 & \multirow[t]{5}{*}{0,0670} & \multirow[t]{5}{*}{1,0000} & \multirow[t]{5}{*}{1} \\
\hline 2 & 0,1727 & 0,1492 & 0,8395 & 0,7419 & 0,2649 & & & \\
\hline 3 & 0,1444 & 0,1492 & 0,6990 & 0,6267 & 0,2613 & & & \\
\hline 4 & 0,1147 & 0,1492 & 0,5665 & 0,5180 & 0,2576 & & & \\
\hline 5 & 0,0837 & 0,1492 & 0,4425 & 0,4167 & 0,2537 & & & \\
\hline 6 & 0,0518 & 0,0701 & 0,5770 & 0,5490 & 0,3960 & \multirow[t]{6}{*}{0,1427} & \multirow[t]{6}{*}{0,3284} & \multirow[t]{6}{*}{2} \\
\hline 7 & 0,0254 & 0,0701 & 0,3897 & 0,3955 & 0,3934 & & & \\
\hline 8 & 0,0100 & 0,0701 & 0,5747 & 0,5449 & 0,3909 & & & \\
\hline 9 & 0,0321 & 0,0701 & 0,7385 & 0,6785 & 0,3886 & & & \\
\hline 10 & 0,0498 & 0,0701 & 0,8806 & 0,7947 & 0,3865 & & & \\
\hline 11 & 0,0675 & 0,0701 & 1,0000 & 0,8926 & 0,3847 & & & \\
\hline 12 & 0,0859 & 0,0970 & 0,8498 & 0,7560 & 0,3125 & \multirow[t]{8}{*}{0,1031} & \multirow[t]{8}{*}{1,0000} & \multirow[t]{8}{*}{3} \\
\hline 13 & 0,0998 & 0,0970 & 0,9046 & 0,8009 & 0,3111 & & & \\
\hline 14 & 0,1091 & 0,0970 & 0,9413 & 0,8309 & 0,3102 & & & \\
\hline 15 & 0,1138 & 0,0970 & 0,9597 & 0,8460 & 0,3098 & & & \\
\hline 16 & 0,1138 & 0,0970 & 0,9597 & 0,8460 & 0,3098 & & & \\
\hline 17 & 0,1091 & 0,0970 & 0,9413 & 0,8309 & 0,3102 & & & \\
\hline 18 & 0,0998 & 0,0970 & 0,9046 & 0,8009 & 0,3111 & & & \\
\hline 19 & 0,0859 & 0,0970 & 0,8498 & 0,7560 & 0,3125 & & & \\
\hline 20 & 0,0675 & 0,0701 & 1,0000 & 0,8926 & 0,3847 & \multirow[t]{6}{*}{0,1427} & \multirow[t]{6}{*}{0,3284} & \multirow[t]{6}{*}{4} \\
\hline 21 & 0,0498 & 0,0701 & 0,8806 & 0,7947 & 0,3865 & & & \\
\hline 22 & 0,0321 & 0,0701 & 0,7385 & 0,6785 & 0,3886 & & & \\
\hline 23 & 0,0100 & 0,0701 & 0,5747 & 0,5449 & 0,3909 & & & \\
\hline 24 & 0,0254 & 0,0701 & 0,3897 & 0,3955 & 0,3934 & & & \\
\hline 25 & 0,0518 & 0,0701 & 0,5770 & 0,5490 & 0,3960 & & & \\
\hline 26 & 0,0837 & 0,1492 & 0,4425 & 0,4167 & 0,2537 & \multirow[t]{5}{*}{0,0670} & \multirow[t]{5}{*}{1,0000} & \multirow[t]{5}{*}{5} \\
\hline 27 & 0,1147 & 0,1492 & 0,5665 & 0,5180 & 0,2576 & & & \\
\hline 28 & 0,1444 & 0,1492 & 0,6990 & 0,6267 & 0,2613 & & & \\
\hline 29 & 0,1727 & 0,1492 & 0,8395 & 0,7419 & 0,2649 & & & \\
\hline 30 & 0,1995 & 0,1492 & 0,9871 & 0,8633 & 0,2685 & & & \\
\hline
\end{tabular}




\section{Лumepamypa}

1. Abd Elrehim, M.Z. Structural optimization of concrete arch bridges using Genetic Algorithms / M.Z., Abd Elrehim, M.A. Eid, M.G. Sayed // Ain Shams Engineering Journal. - 2019. - Vol. 10. - Iss. 3. - P. 507-516.

2. Stress-oriented structural optimization for frame structures / S. Chai, B. Chen, M. Ji [et al.] // Graphical Models. - 2018. - Vol. 97. - P. 80-88.

3. Khan, W. Structural optimization based on meshless element free Galerkin and level set methods / W. Khan, Sirajul-Islam, B. Ullah // Computer Methods in Applied Mechanics and Engineering. - 2019. - Vol. 344. - P. 144-163.

4. Lagaros, N.S. Life-cycle cost structural design optimization of steel wind towers / N.S. Lagaros, M.G. Karlaftis // Computers \& Structures. - 2016. - Vol. 174. - P. 122-132.

5. Nguyen, L.C. Deeplearning for computational structural optimization / L.C. Nguyen, H. Nguyen-Xuan // ISA Transactions. - 2020. - Vol. 103. - P. 177-191.

6. Papavasileiou, G.S. Earthquake-resistant buildings with steel or composite columns: Comparative assessment using structural optimization / G.S. Papavasileiou, D.C. Charmpis // Journal of Building Engineering. - 2020. - Vol. 27. - 100988.

7. Plocher, J. Review on design and structural optimisation in additive manufacturing: Towards next-generationlightweight structures / J. Plocher, A. Panesar // Materials \& Design. - 2019. - Vol. 183. - 108164.

8. Structural Optimization of an Five Degrees of Freedom (T-3R-T) Robot Manipultor Using Finite Element Analysis / G. Shanmugasundar, R. Sivaramakrishnan, S. Meganathan, S. Balasubramani // Materials Today: Proceedings. - 2019. - Vol. 16, Part 2. - P. 1325-1332.

9. Simonetti, H.L. Smoothing evolutionary structural optimization for structures with displacement or natural frequency constraints / H.L. Simonetti, V.S. Almeida, F. de Assis das Neves // Engineering Structures. - 2018. - Vol. 163. - P. 1-10.

10. Structural optimization of elastic circular arches and design criteria / F. Trentadue, A. Fiore, R. Greco [et al.] // Procedia Manufacturing. - 2020. - Vol. 44. - P. 425-432.

11. Ляхович, Л.С. Особые свойства оптимальных систем и основные направления их реализации в методах расчета сооружений / Л.С. Ляхович. - Томск : Издательство Томского государственного архитектурно-строительного университета, 2009. - 372 с.

12. Ляхович, Л.С. Критерий оценки оптимальных решений при формировании кусочно-постоянных участков полки стержней двутаврового поперечного сечения при ограничениях по устойчивости или на величину первой частоты собственных колебаний / Л.С. Ляхович, П.А. Акимов, Б.А. Тухфатуллин // Вестник Томского государственного архитектурно-строительного университета. - 2020. - Том 22. - № 1. - C. 92-105.

13. Lyakhovich L.S. Assessment of the Proximity of Design to Minimum Material Capacity Solution of Problem of Optimization of the Flange Width of I-Shaped Cross-Section Rods with
Allowance for Stability Constraints or Constraints for the Value of the First Natural Frequency and Strength Requirements / L.S. Lyakhovich, P.A. Akimov, B.A. Tukhfatullin // Международный журнал по расчету гражданских и строительных конструкций. - 2020. - Vol. 16. - Iss. 2. - P. 71-82.

14. Хедли, Дж. Нелинейное и динамическое программирование / Дж. Хедли. - М. : Мир, 1967. - 507 с.

15. Lyakhovich, L.S. Assessment Criteria of Optimal Solutions for Creation of Rods With Piecewise Constant Cross-Sections With Stability Constraints or Constraints for Value of the First Natural Frequency. Part 1: Theoretical Foundations / L.S. Lyakhovich, P.A. Akimov, B.A. Tukhfatullin // International Journal for Computational Civil and Structural Engineering. 2019. - Vol. 15. - Iss. 4. - P. 88-100.

16. Lyakhovich, L.S. Assessment Criteria of Optimal Solutions for Creation of Rods With Piecewise Constant Cross-Sections With Stability Constraints or Constraints for Value of the First Natural Frequency. Part 2: Numerical Examples Foundations / L.S. Lyakhovich, P.A. Akimov, B.A. Tukhfatullin // International Journal for Computational Civil and Structural Engineering. 2019. - Vol. 15. - Iss. 4. - P. 101-110.

\section{References}

1. Abd Elrehim M.Z., Eid M.A., Sayed M.G. Structural optimization of concrete arch bridges using Genetic Algorithms. In: Ain Shams Engineering Journal, 2019, Volume 10, Issue 3, pp. 507-516. (In Engl.)

2. Chai S., Chen B., Ji M., Yang Z., Lau M., Fu X.-M., Liu L. Stress-oriented structural optimization for frame structures. In: Graphical Models, 2018, Volume 97, pp. 80-88. (In Engl.)

3. Khan W., Siraj-ul-Islam, Ullah B. Structural optimization based on meshless element free Galerkin andlevel set methods. In: Computer Methods in Applied Mechanics and Engineering, 2019, Volume 344, pp. 144-163. (In Engl.)

4. Lagaros N.S., Karlaftis M.G. Life-cycle cost structural design optimization of steel wind towers. In: Computers \& Structures, 2016, Volume 174, pp. 122-132. (In Engl.)

5. Nguyen L.C., Nguyen-Xuan H. Deep learning for computational structural optimization. In: ISA Transactions, 2020, Volume 103, pp. 177-191. (In Engl.)

6. Papavasileiou G.S., Charmpis D.C. Earthquake-resistant buildings with steel or composite columns: Comparative assessment using structural optimization. In: Journal of Building Engineering, 2020, Volume 27, 100988. (In Engl.)

7. Plocher J., Panesar A. Review on design and structural optimisation in additive manufacturing: Towards nextgenerationlightweight structures. In: Materials \& Design, 2019, Volume 183, 108164. (In Engl.)

8. Shanmugasundar G., Sivaramakrishnan R., Meganathan S., Balasubramani S. Structural Optimization of an Five Degrees of Freedom (T-3R-T) Robot Manipultor Using Finite Element Analysis. In: Materials Today: Proceedings, 2019, Volume 16, Part 2, pp. 1325-1332. (In Engl.) 
9. Simonetti H.L., Almeida V.S., de Assis das Neves F. Smoothing evolutionary structural optimization for structures with displacement or natural frequency constraints. In: Engineering Structures, 2018, Volume 163, pp. 1-10. (In Engl.)

10. Trentadue F., Fiore A., Greco R., Marano G.C., Lagaros N.D. Structural optimization of elastic circular arches and design criteria. In: Procedia Manufacturing, 2020, Volume 44, pp. 425-432. (In Engl.)

11. Lyakhovich L.S. Osobye svojstva optimal'nyh sistem i osnovnye napravlenija ih realizacii $v$ metodah rascheta sooruzhenij [Special properties of optimal systems and the main directions of their implementation in methods of structural analysis]. Tomsk, Publishing House of Tomsk State University of Architecture and Building Publ., 2009, 372 p (in Russ.).

12. Lyakhovich L.S., Akimov P.A., Tukhfatullin B.A. Kriterij ocenki optimal'nyh reshenij pri formirovanii kusochno-postojannyh uchastkov polki sterzhnej dvutavrovogo poperechnogo sechenija pri ogranichenijah po ustojchivosti ili na velichinu pervoj chastoty sobstvennyh kolebanij [Assessment criterion for optimum design solution of piecewise constant sections in rods of rectangular cross-section with stability of first eigen-frequencylimits]. In: Vestnik Tomskogo gosudarstvennogo arkhitekturno-stroitel'nogo universiteta. Journal of Construction and Architecture, 2020, Vol. 22, Iss.1, pp. 92-105. (in Russ., abstr.in Engl.)
13. Lyakhovich L.S., Akimov P.A., Tukhfatullin B.A. Assessment of the Proximity of Design to Minimum Material Capacity Solution of Problem of Optimization of the Flange Width of I-Shaped Cross-Section Rods with Allowance for Stability Constraints or Constraints for the Value of the First Natural Frequency and Strength Requirements. In: International Journal for Computational Civil and Structural Engineering, 2020, Vol. 16, Iss. 2, pp. 71-82. (in Engl., abstr. in Russ.)

14. Hedli J. Nelinejnoe i dinamicheskoe programmirovanie [Nonlinear and dynamic programming]. Moscow, Mir Publ., 1967,507 p.

15. Lyakhovich L.S., Akimov P.A., Tukhfatullin B.A. Assessment Criteria of Optimal Solutions for Creation of Rods With Piecewise Constant Cross-Sections With Stability Constraints or Constraints for Value of the First Natural Frequency. Part 1: Theoretical Foundations. In: International Journal for Computational Civil and Structural Engineering, 2019, Vol. 15, Iss. 4, pp. 88-100. (in Engl., abstr. in Russ.)

16. Lyakhovich L.S., P.A. Akimov P.A., Tukhfatullin B.A. Assessment Criteria of Optimal Solutions for Creation of Rods With Piecewise Constant Cross-Sections With Stability Constraints or Constraints for Value of the First Natural Frequency. Part 2: Numerical Examples. In: International Journal for Computational Civil and Structural Engineering, 2019, Vol. 15, Iss. 4, pp. 101-110. (in Engl., abstr. in Russ.)

Ляхович Леонид Семенович (Томск). Доктор технических наук, профессор, академик РААСН. Профессор кафедры строительной механики ФГБОУ В0 «Томский государственный архитектурно-строительный университет» (634003, Томск, пл. Соляная, д. 2. ТГАСУ). Эл. почта:lls@tsuab.ru.

Акимов Павел Алексеевич (Москва). Доктор технических наук, профессор, академик РААСН. Временно исполняющий обязанности ректора ФГБОУ В0 «Национальный исследовательский Московский государственный строительный университет», профессор кафедры прикладной математики НИУ МГСУ (129337, г. Москва, Ярославское шоссе, д. 26. МГСУ), профессор кафедры строительной механики ФГБОУ В0 «Томский государственный архитектурно-строительный университет» (634003, Томск, пл. Соляная, д. 2. ТГАСУ), профессор департамента строительства Инженерной академии ФГБОУ В0 «Российский университет дружбы народов» (117198, Москва, ул. Миклухо-Маклая, 6. РУДН). Эл. почта: AkimovPA@mgsu.ru, rector@mgsu.ru.

Тухфатуллин Борис Ахатович (Томск). Кандидат технических наук, доцент. Доцент кафедры строительной механики ФГБОУ В0 «Томский государственный архитектурно-строительный университет» (634003, Томск, пл. Соляная, д. 2. ТГАСУ). Эл. почта: bat9203@gmail.com.

Lyakhovich Leonid S. (Tomsk). Doctor of Technical Sciences, Professor, Academician of RAACS. Professor of the Department of Structural Mechanics at the Tomsk State University of Architecture and Building (2 Solyanaya sq., Tomsk, 634003. TSUAB). E-mail:lls@tsuab.ru.

Akimov Pavel A. (Moscow). Doctor of Technical Sciences, Professor, Academician of RAACS. Acting Rector of the National Research Moscow State University of Civil Engineering, Professor of the Department of Applied Mathematics (26 Yaroslavskoye Highway, Moscow, 129337. MGSU), Professor of the Department of Structural Mechanics at the Tomsk State University of Architecture and Building (2 Solyanaya sq., Tomsk, 634003. TSUAB), Professor of the Department of Construction at the Engineering Academy of the Peoples' Friendship University of Russia (6 Miklukho-Maklaya St, Moscow, 117198. Engineering Academy of RUDN). E-mail: AkimovPA@ mgsu.ru, rector@mgsu.ru.

Tukhfatullin Boris A. (Tomsk). Candidate of Technical Sciences, Associate Professor. Associate Professor of the Department of Structural Mechanics at the Tomsk State University of Architecture and Building (2 Solyanaya sq., Tomsk, 634003. TSUAB). E-mail: bat9203@gmail.com. 